\title{
Research on the Elements of Costumes in Dunhuang Feitian Art
}

\author{
Ruifang Zhang ${ }^{1,2}$ \\ ${ }^{1}$ Weinan Normal University, Weinan, Shaanxi, 714099 \\ ${ }^{2}$ Southeast University, School of Arts, Nanjing, Jiangsu, 210096
}

Keywords: Dunhuang Mogao Grottoes; Flying Art; Costume Elements

\begin{abstract}
Flying in the sky is the god of the sky. The image of the flying sky comes from the ancient Indian mythology. It is the god of the ancient Indian mythology, Gan Dapo and Tinnaro, who can sing and dance, and is the artistic form of Buddhism. When the Buddha speaks the scriptures and finally Nirvana, the flying sky will take off in the air, and its clever use of its own dress and streamers will show the sky and the space change, showing a mysterious picture, and the artistic realm has also been sublimated. Dunhuang Feitian is presented in the form of murals. Its image, style, costumes and styling occupy an important position in Dunhuang art. Dunhuang Feitian's costumes have a certain secularization. The national style and Chinese characteristics are very distinct. Therefore, the study of Dunhuang Feitian art the art of costumes can not only study the aesthetics of the secular people in history, but also have important significance for the modern costume design and the inheritance and development of regional art. The paper mainly discusses the costume elements in Dunhuang Feitian art.
\end{abstract}

\section{Analysis of the Art of Dunhuang Feitian}

Flying is the two little gods of the ancient Indian mythology, Gan Dapo and Tinnaro. When the Buddha speaks the scriptures and finally Nirvana, these two little gods will take off in the air and play music. It can be seen that the main functions of Feitian include worship. In Buddhism culture, heaven refers not only to heaven and heaven, but also refers to the honor of the gods; Buddhism was introduced to China during the Wei, Jin, Southern and Northern Dynasties, developed to the peak in the Tang Dynasty, exchanged and integrated with Chinese Taoism, and Buddhism Feitian and the Taoist "Feixian" have a fusion of artistic images, but later generations named the flying god in the Buddhist cave murals as "flying sky". After the Han Dynasty, the development of the Silk Road made Dunhuang a commercial and cultural center. Dunhuang Feitian and the grottoes appeared at the same time, and the image of Feitian art was displayed in the form of murals. Whether it was the image, style, clothing or shape of the flying sky, it reflected The uniqueness of art in the Dunhuang art is very prominent, so Feitian art is the symbol of Dunhuang art, and is the representative of Buddhist art, which embodies the essence of Dunhuang art.

\section{The Development of Dunhuang Feitian Art Costume Elements}

The image of flying sky in Dunhuang murals has appeared since the creation of the 16th National Caves until the end of the Yuan Dynasty. The whole development process has gone through thousands of years, the replacement of the dynasties, the development of the economy, the exchange and integration of different regional cultures, and other factors. The posture, costumes and artistic styles of the Feitian image are constantly changing. The costume art of Dunhuang Feitian is constantly changing with the evolution of the image of Feitian. According to its development process, the development of the costume elements of Feitian Art can be divided into three stages: rise, prosperity and decline:

The sixteen countries to the Southern and Northern Dynasties are the rise of flying art. This period can be divided into four periods: Beiliang, Beiwei, Xiwei and Beizhou. The flying sky appeared in the Beiliang period. At this time, the Indian and Western domains were far-reaching, and their image was closer to the Western-style flying. The flying movement was awkward and stiff. 
The flying was expressed by the distortion of the body, and the body turned into a "V" shape. The costume elements are also mostly nationalized costumes of the Western Regions. The upper body is mostly semi-naked, with long skirts and arms wrapped around the streamers. Some flying days will wear Indian-style crowns. In the northern cool period, the flying blobs have round hair or bald heads or wear Indian crowns. The Mogao Grottoes were boldly colored when they were first built. The craftsmen knew little about Buddhist art and foreign art. Therefore, the flying sky showed a rough and simple beauty. . Some of the flying jewels in the Northern Wei Dynasty were worn with jade jewels, some were not bun, some were naked, some were worn, that is, rectangular underwear, covering two squats, chest and left shoulder, growing to the waist, wearing a long skirt, skirt And the streamers flutter with the wind; the flying posture of the flying sky is also more diverse; the influence of the Central Plains culture on the image and costumes of the flying world in the Western Wei Dynasty has been more prominent. In this period, the flying crown wears the crown or the sun and the moon crown, and the first half is bare or only Supported, wearing a pearl necklace on the neck, wearing a long scarf, waist skirt, shoulder straps; In addition, the Western Wei Dynasty also appeared in the Central Plains flying, the head combed double hair, the upper body stood upright, hands are drumming, Dressed in a sheep-neck dress, arms wrapped around a long scarf, and the sky is dotted with countless streams. At this time, the flying sky can reflect the overall dynamic and decorative effects. The form has a strong rhythm and is mostly feminine. Although the Western-style flying image once again appeared in the Northern Zhou Dynasty, the painting method of Dunhuang Feitian has belonged to the category of the Central Plains figure painting. The clothing aspect is more diverse. The upper body is either naked or wearing only the costumes, or the Central Plains robe, or With a coat of arms, a long skirt, a flag-shaped skirt, barefoot. The contrast of the flying colors in the Northern Zhou Dynasty is even stronger, changing the simple and rough form of the Western Region.

During the Sui and Tang Dynasties, Dunhuang Feitian developed to its peak. At this time, there were not only many types of flying images, but also the most abundant gestures and costumes. The image of Feitian was more and more mature. It absorbed and blended the characteristics of the flying landscapes of India and the Western Region, and innovated. The image of the flying Chinese image was gradually formed. The Sui Dynasty was an important stage in the innovation of Dunhuang Feitian. It merged the Central Plains style with the Western Region style. Therefore, the flying face, body shape, costumes and accessories of this period were very diverse, and the face was full or delicate, and the body was strong or slender. In terms of clothing, the upper body is naked or worn, and there are also long-sleeved dresses or sleeveless skirts. In accessories, or with a crown, or baldness, the body naturally stretches, or solo or swarm. It can be said that the diversified artistic style of the dynasty has laid a solid foundation for the development of the Chinese-style art of flying art. The flying sky of the Tang Dynasty reflects the ever-changing dynamic beauty. The early days of the Tang Dynasty were strong and unrestrained. This was inseparable from the political enlightenment, economic development, and policy opening in the early Tang Dynasty. The Feitian posture in the late Tang Dynasty was beautiful. Plump has become thin and simple, and the costumes have also turned into elegant and light, calm and worrying. Sui and Tang Dynasties began to appear in the sky, because there are three forms of wearing, it is also known as; in addition, it is also an indispensable part of the flying costumes. The flying dynamics of the flying sky are more vivid. Sui and Tang Feitian entwined in two arms, forming a circular or elliptical ring behind them, and the remaining streamers were bilaterally symmetrical. The types of dragonfly in the Tang Dynasty are also very rich, and the length is longer than the colorful clouds in the murals, but the end is full of dynamic. In addition to cockroaches and cockroaches, the downloading of Sui and Tang Feitian has also changed significantly, especially in long skirts, which have a significant increase in length and a long back swing. Although the flying skirt is a long skirt with a waist skirt, the skirt is fixed by a streamer, which is not only practical, but also because the ribbon is soft and elegant, it also greatly increases the decorative and aesthetic of the flying skirt. In the Tang Dynasty, the ribbon length is far. Far more than the length of the skirt, it is a fusion of the hustle and bustle as a unified whole. 
From the Five Dynasties to the Yuan Dynasty in 460 AD, it was the decline period of Dunhuang Feitian. The flying days of this period were mainly imitating the Feitian in the Tang Dynasty. No matter the shape or the dynamics, there was no innovation. The artistic life of Dunhuang Feitian also entered a period of decline. . During the Five Dynasties and Northern Song Dynasty, Feitian mainly inherited the Tang Dynasty, but due to the turbulent political environment and the backward economic environment, the flying days of this period only imitated the Feitian in the Tang Dynasty, but did not inherit the cheerful and vivid image of the Tang Dynasty. Of course, during the Five Dynasties and the Northern Song Dynasty, the Hexi area was under the jurisdiction of the Cao regime. Because of its worship of Buddhism, it built and repaired a large number of caves in the Mogao Grottoes and Yulin Grottoes, hired famous painters and painters at that time, and painted a large number of murals. Yulin Grottoes and Mogao Grottoes also left some flying works with high artistic value, such as Cave 16 of Yulin Grottoes and Cave 327 of Mogao Grottoes. In the Yuan Dynasty, there were few flying images, but the rare ones were preserved. However, from the analysis of a small number of existing data, the original painting style of the Yuan Dynasty was prominent, the composition was full and dense, and the "iron wire" and "hairspring" were used. The drawing method and the coloring is elegant, reflecting the style of the Tang and Song Dynasties.

\section{The Artistic Aesthetic Analysis of Feitian Costumes}

The human body is the eternal theme in the art of human art. The human body is the foundation of creation in any period and in any field. Ancient Greek philosophers believe that the human body is a highly sophisticated organism, a spirit of all things, and artistic creation. The similarity has prompted China's creation of the image of Feitian to be dominated by the human body. To a certain extent, Dunhuang Feitian has brought the combination of body shape and clothing to a perfect state. Only when the clothing is applied to the human body can it reflect its artistic value and practical value. For example, during the period from the 16th to the Northern and Southern Dynasties, Feitian Art was just interested. At this time, the characters were slightly longer and fuller, the limbs were strong and the body length ratio was not coordinated enough. At this time, the flying costumes were mostly widened, and the role of the costumes was appropriate. The problem of uncoordinated proportion of flying bodies is blended. Feitian's costume changes and physical changes have always tended to be the same. For example, the Tang Dynasty was full and graceful, and the flying costumes of the Tang Dynasty were also widened and lengthened, finally achieving the perfect combination of form and costume. It can be seen from this that whether the form or the costume is to express the same artistic theme and coordinate with each other in the same artistic style.

Feitian art takes flight sense as its main form of expression, so "flying" is the soul of flying. The early flying days mainly imitated the flying of the Western Regions. The flying was mainly presented through the distorted posture, and the various shapes were expressed through the various shades of light and dark. People can only see the flying posture in general, lacking the charm of the later flying and flowing. . With the development of Feitian art, its styling means adopts smooth lines, which vividly outlines the flying and light body, which makes the overall shape body appear dynamic, and the fluttering skirt in the costume makes the flying flight appear lighter and more comfortable. . During the Tang Dynasty, the art of flying sky reached its peak, the lines were more rhythmic, the posture of the streamers was more dynamic and changeable, and the characters were more elegant and high-spirited. At this time, the characteristics of flying art were more prominent, realizing the traditional Chinese aesthetics and national characteristics. The organic fusion gives the aesthetic connotation of the artistic image of Feitian, and the dance dress and the dance pose are quite interesting, which makes people feel beautiful. The dance costumes of the Tang Dynasty pursue novelty. For example, the costumes of "Sheng Shou Le" are embroidered with a large group of flowers. This embroidered garment is surrounded by a short blouse, and the blouse is the same color as the embroidered garment. The reason why Feitian can fly without wings is mainly the role of long skirts and dragonflies, while the long skirts and dragonflies can create a flying effect, mainly because the fabrics are soft, light, delicate and smooth. The poet used the phrase "if nothing, really smoke" to describe the clothing fabric of the Tang Dynasty women's clothing, the leno is 
woven from silk, the breathability is very good, there is a looming beauty. The silk fabric is light and flexible, and can dance in the wind and flutter, so the flying sky can look elegant and ethereal.

\section{Conclusion}

In summary, Feitian is an organic integration of diverse cultures, including China's Central Plains culture, Western culture, Indian culture, etc., and the flying force is mainly expressed through physical dynamics, costumes, pipa, colorful clouds and other elements. The elements coordinate and cooperate with each other to form a unified whole, with the same momentum, showing the same kind of turning state, and finally making the flying sky show the effect of volley dancing. It can be said that Feitian is an artistic image bred by the spirit of Chinese national culture, and its artistic image has constantly changed with the development of the times, and it has become more and more suitable for the aesthetics of people in various periods. In modern design, the designer draws rhythm, rhythm, contrast, unity and other aesthetic elements from the art of flying, and studies the perfect combination of flying shapes and costumes, dynamics and costumes, and analyzes how flying murals can create natural flying through dance techniques and costumes. In short, Feitian art has left us a valuable reference, and contemporary designers will get very useful inspiration and rich nourishment.

\section{References}

[1] Zhou Feng, Zhang Enliang. Reference materials of ancient figures [M]. Shenyang Institute of Arts and Crafts, 1979: 120-131.

[2] Dunhuang Research Institute. Clothing Volume [M]. Hong Kong Commercial Press, 2001: 70-78.

[3] Gao Chunming. A test of Chinese costumes [M]. Hong Kong, The Commercial Press, 2001: 81-90.

[4] Zhou Xun, Gao Chunming. "The Great View of Chinese Ancient Costumes" [M]. Chongqing People's Publishing House, 1996: 156-159.

[5] Yoshimura (Japan), Yan Liqiang and other translations. The study of the birth of the heavens the collection of East Asian Buddhist art history [M]. China Federation of Literary and Art Publishing House, 2002: 2745-2747 